\title{
Potential role of flavonoids against SARS-CoV-2 induced diarrhea
}

\author{
Paul, A.K. ${ }^{1,2 *}$, Jahan, R. ${ }^{1}$, Bondhon, T.A. ${ }^{1}$, Jannat, $\mathrm{K}^{1}{ }^{1}$, Hasan, A. ${ }^{1}$, Rahmatullah, M. ${ }^{1}$, Nissapatorn, V. $^{3}$, \\ Pereira, M.L. ${ }^{4}$, Wiart, C. ${ }^{5}$
}

${ }^{1}$ Department of Biotechnology \& Genetic Engineering, University of Development Alternative, Lalmatia, Dhaka-1207, Bangladesh

${ }^{2}$ School of Pharmacy and Pharmacology, University of Tasmania, Hobart, Australia

${ }^{3}$ School of Allied Health Sciences, World Union for Herbal Drug Discovery (WUHeDD), and Research Excellence Center for Innovation and Health Products (RECIHP), Walailak University, Nakhon Si Thammarat 80160, Thailand

${ }^{4} \mathrm{CICECO}$ - Aveiro Institute of Materials and Department of Medical Sciences, University of Aveiro, Aveiro 3810, Portugal

${ }^{5}$ School of Pharmacy, University of Nottingham Malaysia Campus, Semenyih 43500, Selangor Darul Ehsan, Malaysia

*Corresponding author: alok.paul@utas.edu.au

\section{ARTICLE HISTORY}

Received: 2 April 2021

Revised: 26 July 2021

Accepted: 26 July 2021

Published: 30 August 2021

\begin{abstract}
COVID-19, caused by the SARS-CoV-2 virus, can lead to massive inflammation in the gastrointestinal tract causing severe clinical symptoms. SARS-CoV-2 infects lungs after binding its spike proteins with alveolar angiotensin-converting enzyme 2 (ACE2), and it also triggers inflammation in the gastrointestinal tract. SARS-CoV-2 invades the gastrointestinal tract by interacting with Toll-like receptor-4 (TLR4) that induces the expression of ACE2. The influx of ACE2 facilitates cellular binding of more SARS-CoV-2 and causes massive gastrointestinal inflammation leading to diarrhea. Diarrhea prior to COVID-19 infection or COVID-19-induced diarrhea reportedly ends up in a poor prognosis for the patient. Flavonoids are part of traditional remedies for gastrointestinal disorders. Preclinical studies show that flavonoids can prevent infectious diarrhea. Recent studies show flavonoids can inhibit the multiplication of SARS-CoV-2. In combination with vitamin D, flavonoids possibly activate nuclear factor erythroid-derived-2-related factor 2 that downregulates ACE2 expression in cells. We suggest that flavonoids have the potential to prevent SARS-CoV-2 induced diarrhea.
\end{abstract}

Keywords: SARS-CoV-2; diarrhea; flavonoids; quercetin

\section{INTRODUCTION}

Two severe coronavirus incidences emerged before the present pandemic. The first SARS-CoV or Severe Acute Respiratory Syndrome coronavirus was reported from Guangdong Province in China in mid-November 2002 (Wang \& Eaton, 2007). The second, known as Middle East Respiratory Syndrome coronavirus or MERS-CoV was reported from the Middle East in 2012 (Sharif-Yakan \& Kanj, 2014). The third coronavirus, SARS-CoV-2, which is the cause of the present pandemic, was reported from Wuhan, China at the tail end of December 2019 (da Costa et al., 2020). Four other coronaviruses are known to affect humans, namely HCoV229E, HCoV-NL63, HCoV-OC43 and HKU1, but they cause mild upper respiratory tract diseases, which rarely progress to any serious conditions and fatalities (Liu et al., 2021a).

COVID-19, caused by the severe acute respiratory syndrome (SARS)-CoV-2 has led to the largest pandemic of the $21^{\text {st }}$ century. As of July 25,2021 , the recorded COVID-19 cases are $194,583,817$ that caused 4,171,749 deaths globally (https://www.worldometers.info/coronavirus/). To date, at least six vaccines have received emergency approval, but there is significant concern on the safety, efficacy, and feasibility of vaccinating twice approximately 7.8 billion people worldwide. There is also concern about the acceptability of new vaccines, as recent limited surveys showed that not all people wished to vaccinate themselves against SARS-CoV-2 (Dodd et al., 2021). Moreover, COVID-19 vaccines will provide preventative measures against SARSCoV-2 for a limited time.

\section{COVID-19 and diarrhea}

Diarrhea appears to be a common feature in a certain percentage of infected patients, and this has been observed in SARS, MERS, and SARS-CoV-2 infections. Around 10.6 percent of patients reportedly had diarrhea during SARS infection; the percentage rose to $30 \%$ during MERS infection, the latter being much more deadly than SARS (D'Amico et al., 2020).

The COVID-19 induced gastrointestinal manifestations share similar viral or bacterial infection pathways in humans. Briefly, the virus or viral RNAs triggers the body's immune system that activates rapidly to prevent viral antigens and their multiplications inside the body. SARS-CoV-2 infects human lungs after binding its spike proteins with alveolar angiotensin-converting enzyme 2 (ACE2) and causes an increase of angiotensin II in the bloodstream that triggers 
the release of chemokines and activates both cytotoxic $(\mathrm{CD} 8+)$ and helper (CD4+) T-cell lymphocytes and activity of macrophages and NK cells (Ye et al., 2020). Cytotoxic T-cells produce IL-2 and IFN $\gamma$, and helper T-cells release proinflammatory cytokines such as IL-4, IL-17, IL-21, and IFN $\gamma$. It causes massive mucus release and severe acute respiratory distress syndrome. The increased cytokines in the bloodstream and SARS-CoV-2 invasion in the gastrointestinal tract by interacting with gut ACE2 receptors cause gastrointestinal dysbiosis. SARS-CoV-2 also binds with Toll-like receptors (TLR) (especially with TLR4). A healthy gut contains reduced TLR4, but increased expression of TLR4 has been reported under inflammatory conditions (Dheer et al., 2016; Hug et al., 2018). SARS-CoV-2 Spike protein binding with TLR4 may cause increased expression of ACE-II receptors in alveoli's cellular surfaces and thus increase the intensity of infection (Aboudounya \& Heads, 2021). Increased inflammation causes significant damage in the single-cell layers of intestinal mucosa and activations of macrophages, NK-cell, and T-cell lymphocytes to release a massive amount of proinflammatory cytokines and chemokines. The imbalance in the gut microbiota and damage of mucosal cellular barriers facilitate multiplications of SARS-CoV-2 and translocation into other tissues. This dysbiosis triggers diarrhea, bloody diarrhea, mucus secretions, and gastrointestinal pain (Figure 1).

Interestingly, an analysis of COVID-19 severity found a more significant percentage of diarrheal occurrence in severe patients than in less severe patients $(5.8 \%$ versus $3.5 \%$, respectively) (Guan et al., 2020). The opposite also holds true; patients with diarrhea followed by infection with the SARS-CoV-2 virus were more likely to develop acute respiratory distress and require mechanical ventilation than COVID-19 non-diarrheal patients (6.76\% versus $2.08 \%$, respectively) (Jin et al., 2020). This would suggest a gut-lung axis where changes in the gut biome because of COVID-19 would trigger a systemic cytokine release, which may exacerbate COVID-19 induced lung distress. On the other hand, COVID-19 does cause changes in the gut microbiota with a decrease in beneficial microorganisms and an increase in opportunistic pathogens, which can be discerned in the fecal samples of COVID-19 patients (Zuo et al., 2020).

Age, mode of delivery (normal or Caesarian), consumption of nutrients, antioxidants, natural antimicrobial compounds, and probiotics, along with mental well-being, can contribute to gastrointestinal microbiota (Thursby \& Juge, 2017), preventing diarrhea and increase immunity against common viral and bacterial infections. Flavonoids are secondary metabolites of plants. Flavonoids such as quercetin, hesperetin, baicalin, luteolin, gallocatechin and epigallocatechin gallate are antioxidants, anti-inflammatory, and antimicrobial agents. These compounds can boost the immunity of people against viruses as they inhibit some enzymes (e.g. Papain Like protease (PLpro), RNAdependent RNA polymerase (RdRp), Chymotrypsin-Like Protease (3CLpro), and NTPase/helicase), which are essential for the replication and transcription of SARS-CoV-2 (Russo et al., 2020; Mouffouk et al., 2021). Flavonoids like epigallocatechin-3-gallate (from green tea) along with vitamin D3 consumption are believed to activate the nuclear factor erythroid-derived 2-related factor 2 (Nrf2 transcription factor) that down-regulates ACE2 expression from the cellular surfaces and may protect cells against SARS-CoV-2 infection (Mendonca \& Soliman, 2020). This interaction would help develop immunity against infections, reduce oxidative stress, and release proinflammatory cytokines caused by the virus (Figure 1).

\section{Role of flavonoids against COVID-19 induced diarrhea}

Flavonoids can act against COVID-19 induced diarrhea in three ways. It can alleviate COVID-19, it can alleviate diarrhea, or it can alleviate both COVID-19 and diarrhea. Amelioration of acute and chronic diarrhea by flavonoids has been attributed to reducing intestinal motility and reducing chronic gut intestinal inflammatory injury (Gálvez et al., 2001). It has been shown repeatedly that various flavonoids can ameliorate diarrhea through a number of mechanisms. For instance, Psidium guajava has been traditionally used to prevent gastrointestinal disorders in many countries, and flavonoids like quercetin (from Psidium guajava) prevent Shigella and Escherichia coli-induced diarrhea (Hirudkar et al., 2020). Incidentally, quercetin may also inhibit viral entry into host cells and in silico studies have shown that the flavonoid can inhibit Mpro of SARS-CoV-2 (Brito et al., 2021). A similar observation is applicable also for baicalein, a flavonoid present in Scutellaria baicalensis. Baicalein has been shown to inhibit SARS-CoV-2 and its 3C-like protease replication in vitro (Liu et al., 2021b), and suppress colonic motility (Kim et al., 2019). In senna extract-induced acute diarrhea in BALB/C mice, flavonoids from Malus pumila leaves lowered diarrhea index and decreased the levels of inflammatory cytokines like interleukin 6 (IL-6), interleukin 12 (IL-12), and tumor necrosis factor- $\alpha$ (TNF- $\alpha$ ). This ameliorating effect was reported to be caused by ten flavonoids, namely rutin, hyperoside, isoquercitrin, taxifolin, quercitrin, hesperidin, myricetin, baicalin, neohesperidin dihydrochalcone and quercetin ( $\mathrm{Yi}$ et al., 2020). Incidentally most of these flavonoids have been variously reported to be able to bind and inhibit various integral proteins of SARS-CoV-2 necessary for viral entry and replication in human cells (Alzaabi et al., 2021). Thus, flavonoids can have a dual effect of alleviating diarrhea through inhibitory effects on causative agents (like SARS-CoV-2) as well as contractile inhibitions (Zhang et al., 2003).

Quercetin, Luteolin and Quercetin 7-rhamnoside showed efficacy against porcine epidemic diarrhea virus (Choi et al., 2009), as these compounds interfere the viral replication at initial stage and thus similarly it can work against SARSCoV-2 induced diarrhoea. In silico studies exhibit that quercetin interacts firmly with SARS-CoV-2 spike proteins (Colunga Biancatelli et al., 2020; Derosa et al., 2021), and the compound showed potent inhibition against 3CLpro and PLpro enzymes of SARS-CoV-2 (Derosa et al., 2021). Noticeably, its efficacy after oral consumption is limited by its poor solubility and bioavailability, which can be improved by developing a complex with phospholipids like lecithin as it was done in a randomized clinical trial (Di Pierro et al., 2021). In this clinical study, daily oral intake of $1 \mathrm{~g}$ quercetin improved symptoms, reduced fatigue, improved appetite and overall health conditions of non-critical COVID-19 patients (Di Pierro et al., 2021). Flavonoids such as quercetin can inhibit interleukin (IL)-6, IL-17, tumor necrosis factor (TNF)- $\alpha$ and other proinflammatory markers from inflamed GI lumen or other organs (especially lung and other parts of the respiratory system) affected by viral or bacterial infections, which are major symptoms of COVID-19 (Huang et al., 2020; Bastaminejad et al., 2021). The compound (quercetin) also displayed efficacy against lung infections (Heinz et al., 2010; Wang et al., 2018).

Nigella sativa oil or extract (key flavonoids: Quercetin, kaempferol and quercitrin) showed efficacy against diarrhea and protective effects on rodents' gastric and cecal tissues (Eida et al., 2015; Toma et al., 2015). Another clinical study showed (ID: NCT04401202 in ClinicalTrials.gov) that oral treatment of essential oil of Nigella sativa $(500 \mathrm{mg} / \mathrm{capsule}$, 


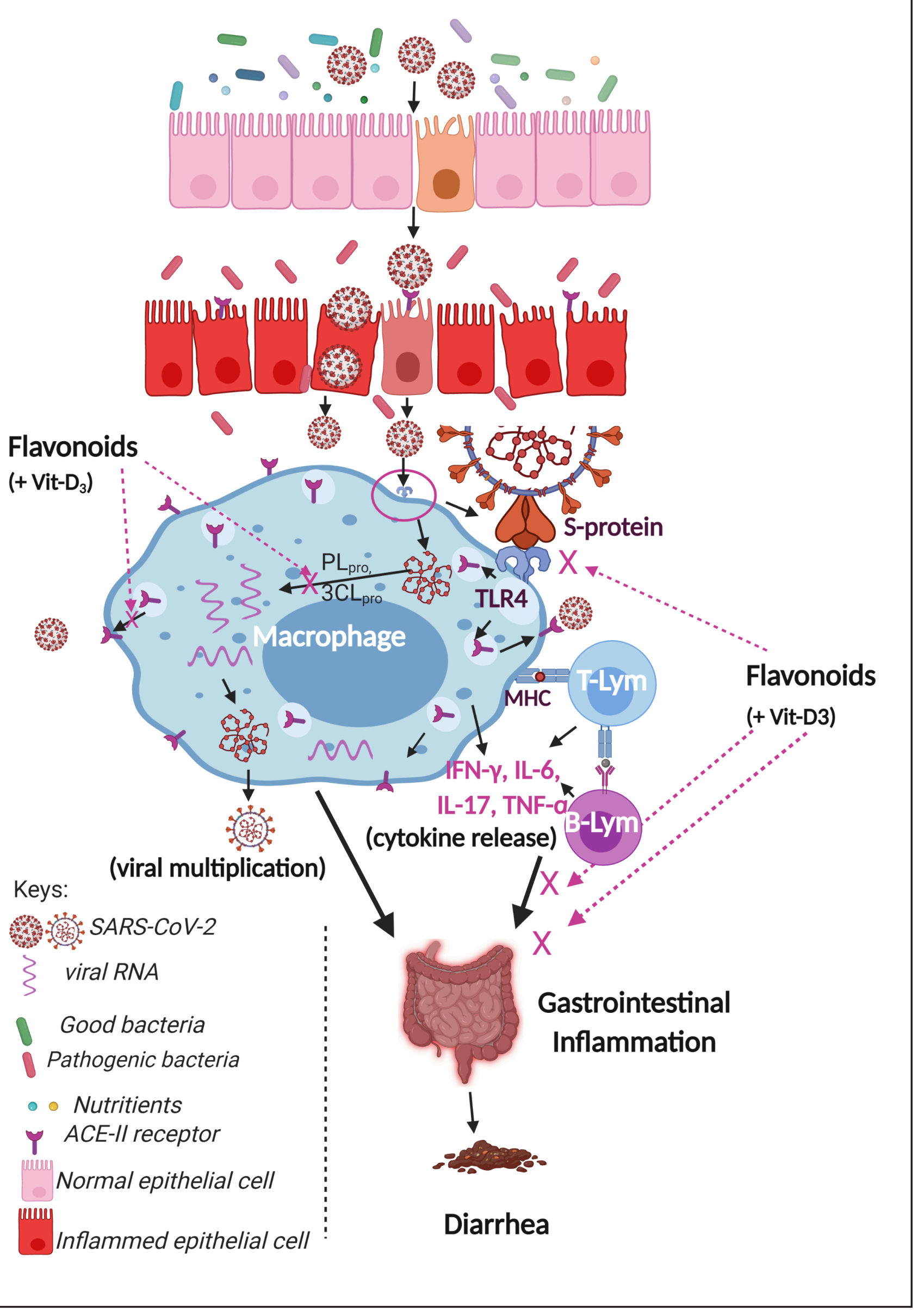

Figure 1. Role of flavonoids against SARS-CoV-2 induced diarrhea (made with biorender.com). 
Table 1. Role of flavonoids against SARS-CoV-2 associated infections

\begin{tabular}{|c|c|c|}
\hline Names of flavonoids & Role against SARS-CoV-2 & Reference \\
\hline $\begin{array}{l}\text { (-)-Epigallocatechin-3-O-gallate } \\
\text { (EGCG) }\end{array}$ & $\begin{array}{l}\text { Inhibited SARS-CoV-2 spike protein interaction with ACE2 } \\
\text { receptor (in silico) }\end{array}$ & (Wang et al., 2021) \\
\hline (-)-Gallocatechin gallate (GCG) & Inhibited $3 \mathrm{CL}_{\text {pro }}$ and $\mathrm{PL}_{\text {pro }}$ proteins (in silico) & (Swargiary et al., 2020) \\
\hline Kaempferol & Inhibited COVID-19 enzymes $3 \mathrm{CL}_{\text {pro }}$ and $\mathrm{PL}_{\text {pro }}$ (in silico) & (He et al., 2020; Khan et al., 2021) \\
\hline Luteolin & $\begin{array}{l}\text { 1. Reduced proinflammatory cytokines } \\
\text { (e.g. TNF- } \alpha, I L-1 \beta, I L-6 \text {, and so on) } \\
\text { 2. Prevented Toll-like receptor } 4 \text { (TLR4)/TNF receptor-associated } \\
\text { factor } 6 / \text { nuclear transcription factor- } K B \text { (NF- } \kappa B \text { ) signaling } \\
\text { pathway of inflammation }\end{array}$ & (Yang et al., 2020) \\
\hline
\end{tabular}

Puerarin

1. Inhibited SARS-CoV-2 spike protein interaction with ACE2 receptor (in silico)

(Qin et al., 2021)

2. It suppressed inflammatory markers (e.g. TNF- $\alpha$, IL-2, IL-17, mitogen-activated protein kinase (MAPK), peroxisome-proliferator activator receptor $\gamma$ (PPAR $\gamma$ ) and nitric oxide synthase 3 (NOS3))

\begin{tabular}{lll}
\hline Quercetin & $\begin{array}{l}\text { Inhibited SARS-CoV-2 spike protein interactions with ACE2 } \\
\text { receptor (in silico) }\end{array}$ & (Di Pierro et al., 2021) \\
\hline Quercetin & Inhibited COVID-19 enzymes $3 \mathrm{CL}_{\text {pro }}$ and $\mathrm{PL}_{\text {pro }}$ (in silico) \\
\hline Quercetin & Reduced proinflammatory cytokines (e.g. IL-6, IL-17, TNF- $\alpha)$ & (Derosa et al., 2021) \\
\hline Luteolin & $\begin{array}{l}\text { Improved smell or taste disorders of patients with post-COVID-19 } \\
\text { infections (clinal study) }\end{array}$ \\
\hline Quercetin & $\begin{array}{l}\text { Quercetin reduced the length of hospital stay and severity of } \\
\text { infections in SARS-CoV-2 infected patients with mild and } \\
\text { moderate symptoms (clinical study) }\end{array}$ \\
\hline
\end{tabular}

twice daily) for ten days moderately improved symptoms of COVID-19 in non-critical patients against patients treated with the standard form of care (Koshak et al., 2020). COVID-19 patients treated with quercetin-rich traditional Chinese herbs reportedly experienced improved immunity against SARS-CoV-2 and less hospitalization (Luo et al., 2020). Various other flavonoids like (-)-Gallocatechin gallate (GCG), (-)Epigallocatechin-3-O-gallate (EGCG), Kaempferol, Luteolin, and Puerarin showed promising effects against COVID-19 infections (Table 1). These flavonoids act against the SARSCoV-2 by three major ways: (1) inhibition of interactions between ACE-2 of host cells and SARS-CoV-2 spike proteins, (ii) inhibition of SARS-CoV-2 protease enzymes like 3CLpro and PLpro and its replication process, and (iii) inhibition of proinflammatory cytokines (e.g. IL-6, IL-17, TNF- $\alpha$ ) caused by SARS-CoV-2 infection (Figure 1, Table 1). SARS-CoV-2 produces inflammation in gastrointestinal tract, lungs and other organs. Flavonoids have antidiarrheal, anti-inflammatory and antiviral properties, as shown in preclinical, preliminary clinical and in silico studies (Colunga Biancatelli et al., 2020; Hirudkar et al., 2020; D’Ascanio et al., 2021; Derosa et al., 2021; Di Pierro et al., 2021). Flavonoids can not only alleviate inflammation through down-regulation of pro-inflammatory cytokines (Ginwala et al., 2019) but also disrupt the lipid raft and inhibit TLR4 signaling (Pei et al., 2020). Thus, flavonoid compounds may provide good therapeutic or supplementary reliefs against SARS-CoV-2 induced diarrhea (Figure 1 ).

To date, any evidence of the role of flavonoids against SARS-CoV-2 induced diarrhea is preliminary, and more importantly, in vitro preclinical and clinical studies are essential to explore the full potential of these compounds against SARS-CoV-2-induced diarrhea. On the other hand, a number of flavonoids have anti-diarrheal effects, as demonstrated in non-COVID-19 patients (Chen et al., 2014). It has been suggested that "flavonoids may ameliorate acute and chronic diarrhea by inhibition of intestinal motility and secretion and may also be helpful in reducing chronic inflammatory injury in the gut by protecting it from oxidative stress and preserving mucosal function" (Galvez et al., 2001). Flavonoids have been used in traditional medicines over the centuries, and these compounds have antimicrobial, antiviral, and gastro-entero-protective effects and may be potential therapeutics for SARS-CoV-2-induced diarrhea. From that viewpoint, flavonoids deserve a closer examination for their potentially beneficial effects in improving COVID-19 prognosis when diarrhea is a comorbidity.

\section{ACKNOWLEDGMENTS}

The work was funded by the authors. No external funding was obtained.

\section{Conflicts of interest}

The authors declare that there are no conflicts of interest and the work has not been submitted elsewhere.

\section{REFERENCES}

Aboudounya, M.M. \& Heads, R.J. (2021). COVID-19 and tolllike receptor 4 (TLR4): SARS-CoV-2 may bind and activate TLR4 to increase ACE2 expression, facilitating entry and causing hyperinflammation. Mediators of Inflammation 2021: 8874339. https://doi.org/10.1155/2021/8874339 
Alzaabi, M.M., Hamdy, R., Ashmawy, N.S., Hamoda, A.M., Alkhayat, F., Khademi, N.N., Joud, S.M.M.A., El-Keblawi, A.A. \& Soliman, S.S.M. (2021). Flavonoids are promising safe therapy against COVID-19. Phytochemistry Reviews 2021: 1-22. https://doi.org/10.1007/s11101-021-09759-z

Bastaminejad, S. \& Bakhtiyari, S. (2021). Quercetin and its relative therapeutic potential against COVID-19: A retrospective review and prospective overview. Current molecular medicine 21: 385-391. https://doi.org/10.2174/ 1566524020999200918150630

Brito, J.C.M., Lima, W.G. \& da Cruz Nizer, W.S. (2021). Quercetin as a potential nutraceutic against coronavirus disease 2019 (COVID-19). Ars Pharmaceutica 62: 85-89.

Chen, Z., Zheng, S., Li, L. \& Jiang, H. (2014). Metabolism of flavonoids in human: A comprehensive review. Current Drug Metabolism 15: 48-61. https://doi.org/10.2174/ 138920021501140218125020

Choi, H.J., Kim, J.H., Lee, C.H., Ahn, Y.J., Song, J.H., Baek, S.H. \& Kwon, D.H. (2009). Antiviral activity of quercetin 7rhamnoside against porcine epidemic diarrhea virus. Antiviral Research 81: 77-81. https://doi.org/10.1016/ j.antiviral.2008.10.002

Colunga Biancatelli, R.M.L., Berrill, M., Catravas, J.D. \& Marik, P.E. (2020). Quercetin and vitamin C: An experimental, synergistic therapy for the prevention and treatment of SARS-CoV-2 related disease (COVID-19). Frontiers in Immunology 11: 1451. https://doi.org/10.3389/fimmu. 2020.01451

D’Amico, F., Baumgart, D.C., Danese, S. \& Peyrin-Beroulet, L. (2020). Diarrhea during COVID-19 infection: Pathogenesis, epidemiology, prevention, and management. Clinical Gastroenterology and Hepatology 18: 1663-1672. https:// doi.org/10.1016/j.cgh.2020.04.001

D’Ascanio, L., Vitelli, F., Cingolani, C., Maranzano, M., Brenner, M.J. \& Di Stadio, A. (2021). Randomized clinical trial "olfactory dysfunction after COVID-19: olfactory rehabilitation therapy vs. intervention treatment with Palmitoylethanolamide and Luteolin": preliminary results. European Review for Medical and Pharmacological Sciences 25: 4156-4162. https://doi.org/10.26355/ eurrev_202106_26059

da Costa, V.G., Moreli, M.L. \& Saivish, M.V. (2020). The emergence of SARS, MERS and novel SARS-2 coronaviruses in the $21^{\text {st }}$ century. Archives of Virology 165:1517-1526. https://doi.org/10.1007/s00705-020-04628-0

Derosa, G., Maffioli, P., D’Angelo, A. \& Di Pierro, F. (2021). A role for quercetin in coronavirus disease 2019 (COVID19). Phytotherapy Research 35: 1230-1236. https://doi.org/ 10.1002/ptr.6887

Dheer, R., Santaolalla, R., Davies, J.M., Lang, J.K., Phillips, M.C., Pastorini, C., Vazquez-Partejo, M.T. \& Abreu, M.T. (2016). Intestinal epithelial toll-like receptor 4 signaling affects epithelial function and colonic microbiota and promotes a risk for transmissible colitis. Infection and Immunity 84: 798-810. https://doi.org/10.1128/iai.01374-15

Di Pierro, F., Khan, A., Bertuccioli, A., Maffioli, P., Derosa, G., Khan, S., Khan, B.A., Nigar, R., Ujjan, I. \& Devrajani, B.R. (2021). Quercetin Phytosome ${ }^{\odot}$ as a potential candidate for managing COVID-19. Minerva Gastroenterology 67: 190195. https://doi.org/10.23736/s1121-421x.20.02771-3

Dodd, R.H., Pickles, K., Nickel, B., Cvejic, E., Ayre, J., Batcup, C., Bonner, C., Copp, T., Cornell, S. \& Dakin, T. (2021). Concerns and motivations about COVID-19 vaccination. The Lancet Infectious Diseases 21: 161-63. https://doi.org/10.1016/s14733099(20)30926-9
Eida, O., Eida, A., Eida, M. \& Dessouki, A.A. (2015). The effect of Nigella sativa aqueous extract on Dientamoeba fragilis: an in vivo experimental study. Parasitologists United Journal 8: 52-59. https://doi.org/10.4103/1687-7942.163409

Galvez, J., Sánchez de Medina, F., Jimenez, J. \& Zarzuelo, A. (2001). Effects of flavonoids on gastrointestinal disorders. Studies in Natural Products Chemistry 25: 607-649. https:// doi.org/10.1016/S1572-5995(01)80019-3

Ginwala, R., Bhavsar, R., Chigbu, D.G.I., Jain, P. \& Khan, Z.K. (2019). Potential role of flavonoids in treating chronic inflammatory diseases with a special focus on the antiinflammatory activity of apigenin. Antioxidants 8: 35. https://doi.org/10.3390/antiox8020035

Guan, W., Ni, Z., Hu, Y., Liang, W., Ou, C., He, J., Liu, L., Shan, H., Lei, C. \& Hui, D.S.C. (2020). Clinical characteristics of coronavirus disease 2019 in China. The New England Journal of Medicine 382: 1708-1720. https://doi.org/10.1056/ NEJMoa2002032

He, T., Qu, R., Qin, C., Wang, Z., Zhang, Y., Shao, X. \& Lu, T. (2020). Potential mechanisms of Chinese Herbal Medicine that implicated in the treatment of COVID-19 related renal injury. Saudi Pharmaceutical Journal 28:1138-1148. https:// doi.org/10.1016/j.jsps.2020.08.002

Heinz, S.A., Henson, D.A., Austin, M.D., Jin, F. \& Nieman, D.C. (2010). Quercetin supplementation and upper respiratory tract infection: A randomized community clinical trial. Pharmacological Research 62: 237-242. https://doi.org/ 10.1016/j.phrs.2010.05.001

Hirudkar, J.R., Parmar, K.M., Prasad, R.S., Sinha, S.K., Lomte, A.D., Itankar, P.R. \& Prasad, S.K. (2020). The antidiarrhoeal evaluation of Psidium guajava L. against enteropathogenic Escherichia coli induced infectious diarrhoea. Journal of Ethnopharmacology 251: 112561. https://doi.org/10.1016/ j.jep.2020.112561

Huang, Y.F., Bai, C., He, F., Xie, Y. \& Zhou, H. (2020). Review on the potential action mechanisms of Chinese medicines in treating Coronavirus Disease 2019 (COVID-19). Pharmacological Research 158: 104939. https://doi.org/10.1016/ j.phrs.2020.104939

Hug, H., Mohajeri, M.H. \& La Fata, G. (2018). Toll-Like Receptors: Regulators of the Immune Response in the Human Gut. Nutrients 10: 203. https://doi.org/10.3390/nu10020203

Jin, X., Lian, J., Hu, J., Gao, J., Zheng, L., Zhang, Y., Hao, S., Jia, H., Cai, H. \& Zhang, X. (2020). Epidemiological, clinical and virological characteristics of 74 cases of coronavirusinfected disease 2019 (COVID-19) with gastrointestinal symptoms. Gut 69: 1002-1009. https://doi.org/10.1136/ gutjnl-2020-320926

Khan, A., Heng, W., Wang, Y., Qiu, J., Wei, X., Peng, S., Saleem, S., Khan, M., Ali, S.S. \& Wei, D.Q. (2021). In silico and in vitro evaluation of kaempferol as a potential inhibitor of the SARS-CoV-2 main protease (3CLpro). Phytotherapy Research 35: 2841-2845. https://doi.org/10.1002/ptr.6998

Kim, H.J., La, J.-H., Kim, H.M., Yang, I.-S. \& Sung, T.S. (2019). Antidiarrheal effect of Scutellaria baicalensis is associated with suppression of smooth muscle in the rat colon. Experimental and Therapeutic Medicine 17: 4748-4756. https://doi.org/10.3892/etm.2019.7469

Koshak, A.E., Koshak, E.A., Mobeireek, A.F., Badawi, M.A., Wali, S.O., Malibary, H.M., Atwah, A.F., Alhamdan, M.M., Almalki, R.A. \& Madani, T.A. (2020). Nigella sativa supplementation to treat symptomatic mild COVID-19: A structured summary of a protocol for a randomised, controlled, clinical trial. Trials 21: 703. https://doi.org/10.1186/s13063-020-04647-x 
Liu, D.X., Liang, J.Q. \& Fung, T.S. (2021a). Human Coronavirus229E, -OC43, -NL63, and -HKU1 (Coronaviridae). Encyclopedia of Virology 2: 428-440. https://doi.org/10.1016/B978-0-12809633-8.21501-X

Liu, H., Ye, F., Sun, Q., Liang, H., Li, C., Li, S., Lu, R., Huang, B., Tan, W. \& Lai, L. (2021b). Scutellaria baicalensis extract and baicalein inhibit replication of SARS-CoV-2 and its 3C-like protease in vitro. Journal of Enzyme Inhibition and Medicinal Chemistry 36: 497-503. https://doi.org/10.1080/ 14756366.2021.1873977

Luo, E., Zhang, D., Luo, H., Liu, B., Zhao, K., Zhao, Y., Bian, Y. \& Wang Y. (2020). Treatment efficacy analysis of traditional Chinese medicine for novel coronavirus pneumonia (COVID-19): an empirical study from Wuhan, Hubei Province, China. Chinese Medicine 15: 34. https://doi.org/ 10.1186/s13020-020-00317-x

Mendonca, P. \& Soliman, K.F.A. (2020). Flavonoids Activation of the Transcription Factor Nrf2 as a hypothesis approach for the prevention and modulation of SARS-CoV-2 infection severity. Antioxidants 9: 659. https://doi.org/ 10.3390/antiox9080659

Mouffouk, C., Mouffouk, S., Mouffouk, S., Hambaba, L. \& Haba, H. (2021). Flavonols as potential antiviral drugs targeting SARS-CoV-2 proteases (3CL(pro) and $\mathrm{PL}($ pro))), spike protein, RNA-dependent RNA polymerase (RdRp) and angiotensinconverting enzyme II receptor (ACE2). European Journal of Pharmacology 891: 173759. https://doi.org/10.1016/ j.ejphar.2020.173759

Pei, R., Liu, X. \& Bolling, B. (2020). Flavonoids and gut health. Current Opinion in Biotechnology 61: 153-159. https://doi.org/ 10.1016/j.copbio.2019.12.018

Qin, X., Huang, C., Wu, K., Li, Y., Liang, X., Su, M. \& Li, R. (2021). Anti-coronavirus disease 2019 (COVID-19) targets and mechanisms of puerarin. Journal of cellular and molecular medicine 25: 677-685. https://doi.org/10.1111/jcmm.16117

Russo, M., Moccia, S., Spagnuolo, C., Tedesco, I. \& Russo, G.L. (2020). Roles of flavonoids against coronavirus infection. Chemico-Biological Interactions 328: 109211. https://doi.org/ 10.1016/j.cbi.2020.109211

Saakre, M., Mathew, D. \& Ravisankar, V. (2021). Perspectives on plant flavonoid quercetin-based drugs for novel SARSCoV-2. Beni-Suef University Journal of Basic and Applied Sciences 10: 21. https://doi.org/10.1186/s43088-021-00107-w

Sharif-Yakan, A. \& Kanj, S.S. (2014). Emergence of MERS-CoV in the Middle East: Origins, transmission, treatment, and perspectives. PLoS Pathogens 10: e1004457. https://doi.org/ 10.1371/journal.ppat.1004457

Swargiary, A., Mahmud, S. \& Saleh, M.A. (2020). Screening of phytochemicals as potent inhibitor of 3-chymotrypsin and papain-like proteases of SARS-CoV2: an in silico approach to combat COVID-19. Journal of Biomolecular Structure \& dynamics 2020: 1-15. https://doi.org/10.1080/07391102. 2020.1835729
Thursby, E. \& Juge, N. (2017). Introduction to the human gut microbiota. The Biochemical Journal 474: 1823-1836. https://doi.org/10.1042/BCJ20160510

Toma, C.C., Olah, N.K., Vlase, L., Mogo'an, C. \& Mocan, A. (2015). Comparative studies on polyphenolic composition, antioxidant and diuretic effects of Nigella sativa L. (Black Cumin) and Nigella damascena L. (Lady-in-a-Mist) Seeds. Molecules 20: 9560-9574. https://doi.org/10.3390/molecules 20069560

Wang, L.F. \& Eaton, B.T. (2007). Bats, civets and the emergence of SARS. Current Topics in Microbiology and Immunology 315: 325-344. https://doi.org/10.1007/978-3-540-70962-6_13

Wang, S., Yao, J., Zhou, B., Yang, J., Chaudry, M.T., Wang, M., Xiao, F., Li, Y. \& Yin, W. (2018). Bacteriostatic effect of quercetin as an antibiotic alternative in vivo and its antibacterial mechanism in vitro. Journal of Food Protection 81: 68-78. https://doi.org/10.4315/0362-028x.Jfp-17-214

Wang, Y.Q., Li, Q.S., Zheng, X.Q., Lu, J.L. \& Liang, Y.R. (2021). Antiviral effects of green tea egcg and its potential application against COVID-19. Molecules 26: 3962. https:// doi.org $/ 10.3390 /$ molecules 26133962

Yang, Y., Tan, X., Xu, J., Wang, T., Liang, T., Xu, X., Ma, C., Xu, Z., Wang, W. \& Li, H. (2020). Luteolin alleviates neuroinflammation via downregulating the TLR4/TRAF6/NF- KB pathway after intracerebral hemorrhage. Biomedicine \& Pharmacotherapy 126: 110044. https://doi.org/10.1016/ j.biopha.2020.110044

Ye, Q., Wang, B., Zhang, T., Xu, J. \& Shang, S. (2020). The mechanism and treatment of gastrointestinal symptoms in patients with COVID-19. American Journal of Physiology Gastrointestinal and Liver Physiology 319: G245-G252. https://doi.org/10.1152/ajpgi.00148.2020

Yi, R., Tian, Y., Tan, F., Li, W., Mu, J., Long, X., Pan, Y. \& Zhao, X. (2020). Intervention effect of Malus pumila leaf flavonoids on senna-induced acute diarrhea in BALB/c mice. Food Science \& Nutrition 8: 2535-2542. https://doi.org/10.1002/ fsn3.1549

Zhang, W.-j., Chen, B.-t., Wang, C.-y., Zhu, Q.-h. \& Mo, Z.-x. (2003). Mechanism of quercetin as an antidiarrheal agent. Di $Y i$ Jun Yi Da Xue Xue Bao 23: 1029-1031.

Zuo, T., Zhang, F., Lui, G.C.Y., Yeoh, Y.K., Li, A.Y.L., Zhan, H., Wan, Y., Chung, A.C.K., Cheung, C.P. \& Chen, N. (2020). Alterations in gut microbiota of patients with COVID-19 during time of hospitalization. Gastroenterology 159: 944-955. https:// doi.org/10.1053/j.gastro.2020.05.048 